HUMANIDADES Y CIENCIAS SOCIALES

INVESTIGACIÓN

\title{
Pragmáticas, dóciles, cuestionadoras: la pregunta por la agencia en usuarias de TRHA
}

Johnson, María Cecilia*

\section{Resumen}

Este trabajo explora las maneras en que las mujeres usuarias de tecnología reproductiva en Argentina construyen diversas formas de agencia a lo largo de sus itinerarios reproductivos. Las tecnologías reproductivas forman parte de los procesos de control y medicalización creciente sobre la reproducción y la sexualidad, aunque paradójicamente este mismo dispositivo biomédico es presentado en nuestra sociedad como la posibilidad más simple para que las mujeres accedan a la maternidad. Esta tensión que algunos sectores académicos advierten sobre la tecnología reproductiva se expresa en las experiencias de las usuarias en diferentes formas de agencia, las cuales amplifican la idea de agencia como resistencia a la biomedicina. Desde un enfoque biográfico se analizan las experiencias de las usuarias y se proponen diversas categorías a partir del trabajo de investigación para comprender la complejidad de la agencia reproductiva de las usuarias.

Palabras clave: reproducción asistida; agencia; resistencia; feminismo

El artículo parte del trabajo doctoral de tesis Nuevas tecnologías reproductivas: sentidos sobre el parentesco en las trayectorias biográficas de mujeres de Córdoba realizada en el contexto del Centro de Estudios Avanzados (UNC), financiada con una beca doctoral otorgada por el Consejo Nacional de Investigaciones Científicas y Técnicas (CONICET). Recibido el 07/02/2020 y aceptado el 07/09/2020.

DOI: https://doi.org/10.33255/3161/725

Autoría: CIECS-CONICET. Universidad Nacional de Córdoba (Argentina).

Contacto: cecilia.johnson@unc.edu.ar 


\title{
Pragmatic, docile, questioning: the question by the agency in users of ARTs
}

\begin{abstract}
This paper explores how women users of reproductive technology in Argentina build agency at different forms along their reproductive itineraries. Reproductive technologies are part of the processes of control and increasing medicalization on reproduction and sexuality, although paradoxically this same biomedical device is presented in our society as the simplest possibility for women to access motherhood. This tension that some academic sectors warn about reproductive technology is expressed in the experiences of users in different forms of agency, which amplifies the idea of agency as resistance to biomedicine. From a biographical approach, user experiences are analyzed, and various categories are proposed from the research work to understand the complexity of the users' reproductive agency.
\end{abstract}

Keywords: Assisted Reproduction; Agency; Resistence; Feminism

\section{Pragmáticas, dóceis, questionadoras: a pergunta sobre a agência em usuárias de TRHA}

\section{Resumo}

Este trabalho explora as maneiras em que as mulheres usuárias de tecnologia reprodutiva na Argentina constroem diversas formas de agência ao longo de seus itinerários reprodutivos. As tecnologias reprodutivas fazem parte dos processos crescentes de controle e medicalização da reprodução e a sexualidade, embora, paradoxalmente, esse mesmo dispositivo biomédico se apresente em nossa sociedade como a possibilidade mais simples para as mulheres terem acesso à maternidade. Esta tensão que alguns setores académicos observam sobre a tecnologia reprodutiva é expressa nas experiências das usuárias em diferentes formas de agência, que amplificam a ideia de agência como resistência à biomedicina. A partir de uma abordagem biográfica são analisadas as experiências das usuárias e são propostas diversas categorias com base no trabalho de pesquisa, para compreender a complexidade da agência reprodutiva das usuárias.

Palavras-chave: reprodução assistida; agência; resistência; feminismo 


\section{Introducción}

Estudiar las experiencias con las TRHA (Técnicas de Reproducción Humana Asistida) supone partir de una tensión que se presenta irreconciliable: el mismo escenario biomédico donde las mujeres experimentan diferentes formas de control y la medicalización de su reproducción y sexualidad constituye paradójicamente el principal recurso con el que cuentan para resolver sus obstáculos para ser madres. De esta manera, en nuestra sociedad argentina, la vía de resolución para la llamada infertilidadํque se encuentra disponible -y que además promete ser sencilla y rápida- es la vía patologizante y medicalizada. Esta solución les exige a las usuarias poner el cuerpo para agenciar su decisión, operación que al mismo tiempo medicaliza la búsqueda de un embarazo desde un paradigma de la infertilidad que invisibiliza el acceso de usuarias por fuera de arreglos heteronormados. Siguiendo a Charis Thompson (2005), este trabajo parte de constatar, entonces, que existen procesos de creciente medicalización y control de los cuerpos gestantes en el proceso de tratamiento con las TRHA. Sin embargo, junto con ello se advierte la convivencia de procesos de agencia en las personas usuarias de TRHA, es decir, estos procesos no son incompatibles (Thompson, 2005: 185).

En el marco de estas discusiones, este artículo analiza las formas de agencia identificadas en los itinerarios de usuarias de TRHA en Córdoba, Argentina. Aquí se presentan algunos resultados de un trabajo de investigación doctoral en estudios de género. El proceso de investigación se centró en relatos biográficos de usuarias que planteaban con gran determinación la manera en que llevaron adelante tratamientos -en ocasiones sucesivos- con el objetivo de tener hijos. Nos encontramos con relatos ambivalentes: usuarias que comparten su experiencia reproductiva atravesada por obstáculos de distinta índole, pero al mismo tiempo relatan situaciones atravesadas por innumerables estrategias que les permitieron llevar adelante el tratamiento.

Este conjunto de formas de agencia se corresponde a su vez con las características propias del tratamiento de TRHA. Como señala Charis Thompson (2005), la coreografía ontológica de las TRHA supone la coordinación de múltiples temporalidades, el ciclo menstrual y el calendario laboral, sin perder de vista el llamado «reloj biológico», interiorizado por las usuarias como una variable determinante. Ello supone un entramado muy complejo donde la resistencia y oposición al sistema biomédico no constituye, de acuerdo a las experiencias, la mejor estrategia para realizar el tratamiento. Este trabajo presenta diversas formas de comprender la agencia de las usuarias, como disposiciones que se van transformando de acuerdo a las acumulaciones, los 
aprendizajes y la apropiación de la tecnología reproductiva a lo largo del tratamiento.

\section{Itinerarios y formas de agencia en usuarias de TRHA}

Para comprender la experiencia de las usuarias de TRHA fue preciso recurrir a diversas tradiciones teóricas que permitieran, a su vez, aproximarse a los sentidos, posibilidades y limitaciones de su acción en el marco del proceso de medicalización de su reproducción. Comprender la agencia de la usuarias en el marco de un dispositivo de gobernabilidad reproductiva (Armstrong, 1995; Morgan, 2019; Rose, 2007) lleva a que en este apartado se expliciten un conjunto de decisiones teóricas que se presentan como ejes de discusión que atraviesan el análisis de las narrativas. El primer eje se vincula a la elección de un punto de vista centrado en la experiencia de las mujeres usuarias a lo largo de los itinerarios, como proceso de medicalización de su reproducción (Peter Conrad, 1990).

Desde hace algunas décadas, diversos estudios se han centrado en el punto de vista de los sujetos retomando sus experiencias y formas de agencia en la atención de su proceso de salud-enfermedad (Mercado-Martínez et al., 1999). Resulta un antecedente central el trabajo de Erving Goffman (2001) con el análisis de la carrera del paciente psiquiátrico, porque permitió situar las transformaciones subjetivas e identitarias en los sujetos en las instituciones psiquiátricas. Asimismo, existe una abundante producción teórica que analiza las propias vivencias y estrategias de los sujetos con enfermedades crónicas o de larga duración, retomando diferentes instancias de sus trayectorias. Michael Bury (1982), desde la noción de «disrupción biográfica», permite explicar cómo el diagnostico de una enfermedad supone un acontecimiento que modifica la biografía de los sujetos y que trastoca la vida cotidiana (Osorio Carranza, 2017).

Así también, estas líneas de análisis han permitido modificar la mirada de los usuarios del sistema de salud como sujetos pasivos, para pasar a comprenderles como agentes en sus tratamientos, tanto en las formas de autoatención de la salud (Menéndez, 2003), como a partir de advertir las estrategias que los sujetos despliegan para cuidar y atender su salud (Mendes Diz, Domínguez Mon y Schwarz, 2011). Desde el estudio de la biopolítica, Nikolas Rose (2007) comprende estas formas de agencia como aquella disposición activa de los pacientes que precisa el campo biomédico, que habilita procesos de «ciudadanía biológica» como forma de acceso a los servicios de salud, perspectiva relevante en este estudio para comprender los procesos de salud reproductiva que por momentos supone asumirse como «ciudadanas biológicas» que agencian por sí mismas los procesos de atención de la salud reproductiva. 
Desde el estudio de las TRHA, Charis Thompson (2005) destaca la importancia de entender la propia coreografía que implica el tratamiento de las TRHA en el marco de estos itinerarios biomédicos. En Argentina, algunas autoras también han estudiado las TRHA desde las propias narrativas e itinerarios de las usuarias (Ariza, 2014; Straw, 2013; Libson, 2012) como un aspecto central para comprender las transformaciones subjetivas.

Estos trabajos constituyen antecedentes centrales dado que los tratamientos de reproducción asistida acontecen en el marco de un campo biomédico que presenta sus reglas: existe un marco normativo y médico que predefine algunos recorridos por técnicas de diferente complejidad, vinculado a un diagnóstico médico que va intentando diferentes alternativas, respondiendo a las formas disponibles de cobertura y los protocolos de las clínicas en Argentina. Este marco coexiste con la singularidad de las experiencias de las mujeres usuarias en su trayectoria: por los recursos culturales y económicos con los que cuentan, por las diferentes estrategias que se establecen con los médicos y con las clínicas, todos aspectos que dan cuenta de los márgenes de agencia posibles en este campo biomédico.

La reconstrucción de estos itinerarios lleva a un segundo eje de análisis que atraviesa este trabajo: la pregunta por las posibilidades de agencia de las usuarias en el marco de esta «carrera» como usuarias. Desde los estudios feministas se sostienen diversas posturas críticas sobre las TRHA entendiendo que estas contribuyen a la reproducción del mandato patriarcal que presenta a la maternidad como el destino inexorable para las mujeres (Corea, 1985; Crowe, 1985; Stolcke, 1986; Waldby y Cooper, 2010). Sin embargo, como señala Franklin (2009), fue a partir del análisis de las experiencias de las usuarias cuando comenzaron a advertir que algunas de estas posiciones se sustentaban ignorando la mirada de estas sobre los mismos, incorporándose su mirada como agentes en diversos estudios feministas en el campo de la salud. La contracara de ello en la reproducción asistida supone lo que Mariana Viera Cherro (2015) critica como una «nueva virtud» de las usuarias: se espera que sean agentes activos en su tratamiento, lo que de alguna manera reactualiza un mandato muy antiguo que supone que las mujeres son reproductoras por definición. Lo mismo se expresa, señala la autora, en el desarrollo mismo de las TRHA:

El desarrollo biotecnológico se orienta en su investigación y práctica, y es acompañado por una dimensión cultural que avala tal sentido, a intervenir mayormente el cuerpo femenino, y no solamente el de la mujer que busca tener descendencia mediante TRHA sino también el de aquellas mujeres que comercian sus óvulos para estos tratamientos (Viera Cherro, 2015: 364). 
Un tercer eje de discusión que recorre este trabajo se vincula a la pregunta por la definición misma de la agencia de las usuarias en el marco de la reproducción asistida. Si bien las TRHA se practican en Argentina desde hace más de treinta años y desde 2012 se encuentran reconocido su acceso como derecho reproductivo, este reconocimiento legal no se traduce en una autonomía total de las usuarias para decidir sobre su reproducción, pero tampoco en una falta de ella.

En el presente análisis se parte de comprender que la relación de las usuarias de TRHA con el poder biomédico es dinámico, a diferencia de aquellas perspectivas que entienden que el poder se encuentra centralizado en el profesional médico y las usuarias no tienen margen de acción. Es por ello que este estudio implicó desplazarse de una idea binaria del poder que se resiste o al que se muestra sumisión (Mahmood, 2008), para comprender diferentes posibilidades de acción, que en este caso es posible de diferentes maneras de acuerdo al momento del tratamiento que estén transitando las usuarias.

Para comprender esta dimensión decisional se parte de un análisis situado de sus decisiones. En esta línea de análisis se destaca el desarrollo de Luc Boltanski y Laurent Thévenot (2000), quienes proponen un sentido situado de la justicia. Para los autores los sujetos aplican las reglas de forma situada, tomando como referencia las propias circunstancias en donde se realiza este juicio de valor o decisión. Mirando los procesos reproductivos, estas decisiones situadas permiten explicar la agencia de las usuarias en el marco de situaciones reproductivas dilemáticas, en donde las usuarias pueden ser descriptas como «pioneras morales» al decir de Rayna Rapp (1999).

Sin embargo, si bien se coincide con la mirada situada que proponen dichos autores, en las decisiones reproductivas cotidianas las practicas de las usuarias se asemejan más a lo que Pierre Bourdieu (2005) denomina «sentido práctico» por su carácter de disposición internalizada para actuar. Esta noción es útil para analizar la agencia reproductiva porque permite comprender que la reproducción se produce en un orden de reflexividad que resulta ajena al cálculo racional y que se expresa en lo discursivo como cierto «pragmatismo» en las decisiones reproductivas de usuarias de TRHA, como se abordará en este trabajo.

Aquellos momentos del itinerario de las usuarias asociados a una fuerte intervención tecnológica en la reproducción y los cuerpos de estas son aquellos que en mayor medida desafían el presente análisis. A priori pareciera una contracción hablar de agencia en el marco de estos procesos de medicalización, ya que a simple vista se advierte sujeción, como señalan algunas 
posturas feministas. Sin embargo, desde una perspectiva foucaultiana que entiende el poder como disciplinante pero al mismo tiempo es constructor de ciertas habilidades, es posible comprender esta ecuación de otra manera. Retomando la perspectiva sobre el poder de Saba Mahmood (2001, 2008), crítica de las conceptualizaciones occidentales que entienden la agencia siempre como oposición a la norma, se entiende que las personas pueden habitar las normas y no necesariamente resistirse a ellas. Retomando el concepto de Orit Avishai (2008) para comprender la agencia de las mujeres en las religiones ortodoxas, se denominará como «agencia dócil» a una manera de entender la disposición de las usuarias en aquellas experiencias relatadas como dolor, incomodidad, disciplina y fortaleza a la hora de iniciar y sostener el tratamiento. Así mismo, estas formas de agencia desde la propia ambivalencia son las que permiten a las usuarias «gestionar sus emociones» en un itinerario que les demanda sostener procesos de pérdida y momentos de fracaso del tratamiento. Para ello, el aporte del llamado «giro afectivo» y la propuesta de Sara Ahmed (2015) subyacen los análisis aquí presentados y permiten analizar las formas en que las usuarias deben aprender a modular y equilibrar sus experiencias emocionales, aspecto que es disciplinante pero a la vez condición necesaria para continuar con el tratamiento.

En esta línea, Saba Mahmood (2008) señala, siguiendo a Foucault, que el poder no es solo un conjunto de relaciones que dominan al sujeto sino que también otorgan las condiciones para su existencia, aspecto que Judith Butler llamara la paradoja de la subjetivación. Esto es así según la autora:

\footnotetext{
... porque las condiciones y procesos que lo subordinan son los mismos que convierten al sujeto en consciente de sí mismo y en agente social (...) Tal conceptualización del poder y la formación del sujeto nos ayuda a entender la agencia social no simplemente como sinónimo de resistencia a las relaciones de poder, sino como la capacidad de acción que ciertas relaciones específicas de subordinación crean y hacen posible (Mahmood, 2008: 179-180).
}

Este proceso que aparece de forma paradojal permite reflexionar sobre el modo en que se habita el espacio biomédico: el mismo sistema que estigmatiza a las usuarias como infértiles es el espacio donde ellas construyen formas de agencia en un campo complejo, críptico y disciplinante. Como señala Judith Butler (1997) cuando hace referencia al lenguaje de odio: «El nombre por el que se le llama a uno te subordina y te capacita, produciendo una situación de agencia desde la ambivalencia, un conjunto de efectos que exceden las intenciones de la nominación» (Butler, 1997: 261). 
Asimismo, la posibilidad de reapropiarse del lenguaje médico es un aspecto que también ha sido analizado por teóricas feministas de los estudios de ciencia y tecnología. Estos desarrollos comienzan a entender a la biología como un artefacto y un discurso sustentado por el aparato científico (Haraway, 1999; Franklin, 2009; Marylin Strathern, 1992). Ello trae nuevas miradas y comprensiones sobre el cuerpo gestante no como un ente natural diferente a la tecnología, sino como parte de la misma. Un antecedente relevante es el trabajo de Emily Martin (2001), quien puso de relieve la construcción sociopolítica y patriarcal de la sexualidad y la reproducción (Stolcke, 2010). A partir de un análisis pormenorizado de los textos de estudio de la medicina reproductiva, Martin analizó las categorías mediante las cuales se nombra a lo biológico, apuntando que comprender a los óvulos como un «recurso no renovable» o entender la ausencia de un embarazo como un «desperdicio» evidencia una mirada patriarcal. Ello le permitió a la autora advertir en los discursos médicos la reproducción de representaciones fragmentadas y alienadas sobre aquello entendido como meramente biológico, a la vez que reproducen estereotipos patriarcales de género. Al mismo tiempo, estos discursos eran reproducidos por las mujeres, expresando la sensación de enajenación del propio cuerpo, percibiéndolo como un ente-objeto que es animado pero operado por otros, como una máquina, lo que da cuenta de una alienación que De Beauvoir ya señalaba décadas atrás. Retomando las discusiones mencionadas, a lo largo del desarrollo de este trabajo se proponen algunas categorías que permiten comprender los relatos de usuarias y las diferentes formas de agencia que ejercen a lo largo de su itinerario reproductivo. Se analizarán estas formas de agencia como disposiciones y modalidades de acción en el campo biomédico como modos de acción posibles para iniciar, sostener y finalizar el tratamiento con TRHA.

\section{Metodología}

A partir de una metodología cualitativa y a través de un enfoque biográfico, se entrevistó a mujeres (cis) usuarias de técnicas de reproducción asistida (TRHA) 2 de la Provincia de Córdoba, Argentina, en el período 2017-2018. El enfoque biográfico permite un trabajo de metarreflexión que reconoce el papel de analista de las usuarias, por lo que este análisis se sitúa en un segundo nivel de interpretación (Ricoeur en: Cornejo, Mendoza y Rojas, 2008: 30).

Se entrevistó a veinte mujeres usuarias de entre 32 y 65 años, las que se pueden caracterizar casi en su totalidad por su inclusión en sectores medio-altos con estudios terciarios a universitarios. Respecto a sus expe- 
riencias, algunas usuarias lograron un embarazo con las TRHA (11) y otras sin tratamiento (3), algunas se encontraban realizando el tratamiento al momento de la entrevista (2) y otras (4) abandonaron el tratamiento luego de sucesivos intentos 3 . A excepción de un solo caso, la totalidad de las usuarias realizó el tratamiento en un establecimiento privado de reproducción asistida.

Al momento del tratamiento, algunas usuarias se encontraban en pareja con un varón (16), otras (2) en una relación de pareja con una mujer y otras no estaban en pareja sino que transitaron el tratamiento desde la monoparentalidad (2).

En cuanto a la religiosidad, cuatro entrevistadas se identificaron como «no creyentes» o «ateas» y una como «agnóstica», y quince se identificaron como «creyentes». Dentro de esta amplia categoría donde se encuentran las quince usuarias identificadas como «creyentes», diez de ellas se definían como «católicas», una como «católica sincrética», una como «creyente en Dios pero sin religión» y otra como una «budista».

Se realizó en primer lugar un análisis de codificación de las entrevistas de modo singular, recuperando los sentidos de las usuarias sobre la experiencia reproductiva en cada relato de vida. Mediante una segunda codificación, se llevó a cabo una lectura transversal de los relatos, encontrando algunas coincidencias en las lógicas de acción, en las disposiciones y en las experiencias a lo largo del tratamiento, lo que permitió proponer algunas categorías como ejes de análisis, las que se presentan a continuación.

\section{La decisión de iniciar el tratamiento: la agencia pragmática}

Una característica común que las mujeres usuarias relatan sobre el inicio del tratamiento es el pragmatismo con el que afrontan su acceso a las TRHA. Se entiende al pragmatismo como una modalidad de acción, hace referencia al «sentido práctico» (Bourdieu, 2005) con el que se aborda este tratamiento con el objetivo de lograr un embarazo. Asimismo, ello coincide con cierta característica de los procesos y decisiones reproductivos, los cuales operan con una reflexividad que resulta ajena al cálculo racional (Petchesky, 1987; Rostagnol, 2015), como una praxis donde las mujeres identifican ciertas necesidades reproductivas sin desconocer las condiciones objetivas en las que se encuentran.

Este pragmatismo aparece en los relatos de usuarias como una acción que busca sortear con rapidez los obstáculos para tener un bebé, situación que genera angustia y ansiedad en un momento determinado. Como relata Nadia, una de las usuarias entrevistadas, no existe un momento deliberativo 
y racional a la hora de comenzar un tratamiento, sino más bien se accede al mismo como una forma de resolución de esta situación:

... nunca me detuve a pensarlo demasiado, apareció un problema y ya era «a resolverlo», nunca, ni me victimicé porque no podía quedar embarazada, ¿viste? No lo padecí desde ese lugar, sí el anhelo de quedar era como angustiante, pero no... A ver, «hay un quiste, operamos el quiste», aparece otra cosa, se resuelve, hay que hacer un estudio, se hace. En ese sentido te digo esto de ser expeditiva, después el proceso emocional iba por otro lado (Nadia, 50 años).

Otro aspecto asociado con esta disposición pragmática tiene que ver con una reflexividad guiada por el orden de lo emocional, el deseo, que no está necesariamente orientada desde una lógica «racional-intelectual». De alguna manera presenta una dimensión de lo afectivo y de lo emocional que no se manifiesta necesariamente desde una lógica del cálculo. Sin embargo, esta disposición práctica responde a un habitus de género, desde la perspectiva de Bourdieu, una visión y representación incorporada vinculada a la sexualidad, la reproducción y la familia. Así lo expresaba Nadia:

No, no sé. La verdad es que no sé. Y perdón, insisto, creo que cuando el deseo es tan fuerte uno apela a lo que puede, digamos, parece que todo método vale, no sé hasta dónde todo método vale, nunca lo analicé demasiado.

$\mathrm{E}:$ ¿Vos lo decís porque el deseo legitima o lo decís con signo en pregunta? $\mathrm{N}$ : Me lo cuestiono, la verdad es que no sé hasta dónde (...) nunca lo hablé con gente tampoco cercana en el tema. Entonces no sé tampoco como para valorar realmente las emociones, las sensaciones, es muy teórico el análisis que uno puede hace ahora. No sé, la verdad (Nadia, 50 años).

Por otra parte, es tan complejo el tránsito por estos espacios biomédicos, donde está en juego no solo la posibilidad de maternar sino la propia salud de las mujeres, que aunque las usuarias dan cuenta de saberes y un gran volumen de capital cultural acumulado en sus trayectorias, la complejidad de decisiones a tomar precisa de un «salto de fe» y de confianza de parte de la usuarias hacia la medicina.

En este sentido, el costo de este pragmatismo tiene que ver con depositar en una autoridad médica las decisiones, sobre todo en la primera parte del tratamiento, cuando aún se desconoce el proceso. Ello aparece con claridad en el relato de Celeste, quien utiliza el término "autómatas» para hablar de actitud de ella y de su pareja al inicio del tratamiento. Seguir todas las 
indicaciones médicas, si bien permite una gran capacidad de acción y operatividad frente a una situación desconocida, se hace necesario en un escenario que requiere actuar en diferentes vías de forma simultánea: informarse, llevar a cabo el tratamiento, conseguir la cobertura, tomar decisiones:

Estábamos como autómatas, la verdad en esos momentos uno hace cosas que por ahí no está tan consciente (...) el médico nos decía vayan y háganse tal estudio y lo hacíamos, decía vayan y hagan tal cosa y lo hacíamos... (Celeste, 36 años).

Esta disposición pragmática es la que a su vez se precisa para entrar en un orden de decisiones complejas donde las usuarias no disponen de toda la información. En el caso de quienes encontraron el uso de las TRHA de forma dilemática por entrar en conflicto con sus posturas religiosas y morales, hay cierta conciencia de la necesidad de asumir esta disposición subjetiva que vincula con lo que se analizó como «sentido práctico» (Bourdieu, 2005). Así lo cuenta María, quien se autodefine como «católica sincrética» y que luego de un tiempo de deliberación personal por entrar en conflicto con la teología católica, decide comenzar los tratamientos. En sus palabras, significó poder hacer un clic y asumir la actitud que advertía en su marido, más práctica.

Desde otras investigaciones se ha señalado cierta «inercia» que presentan las usuarias de TRHA en sus recorridos. Siguiendo al planteamiento de Ana Gual Martí (2011), existe en algunas mujeres usuarias de TRHA un discurso «inercial-adapatativo», «donde la expectativa social ha sido incorporada» (Gual Martí, 2011: 274). En esta línea, algunas usuarias señalan que la decisión de usar las TRHA formó parte de un repertorio de decisiones que se corresponden con las expectativas de la adultez para las mujeres:

... lo que pasa es que nosotros hicimos todo como muy rápido. Nos casamos, hicimos esto, hicimos, nos aturdimos con cuestiones, y no lo pensamos mucho. Igual, no, yo no lo pensaría mucho tampoco... (Melina, 41 años).

De igual manera comenta Catalina, este pragmatismo también se vincula a aquello que aparece en el orden de lo naturalizado, incorporado, que no precisa ser deliberado y donde las TRHA aparecen como la respuesta médica que se ofrece como resolución del deseo de un/a hija/o en el marco de un proyecto de vida:

Entonces... yo creo que hay gente que ni la piensa, va [haciendo referencia a la acción]. (...) para mí es así, es intrínseco, así como tengo que estudiar una carrera, 
cosas, mandatos, y además la quería, (...) nunca fue «¿hacemos un tratamiento?». No. Era no podemos, y sí, vayamos a consultar un médico, o sea los dos somos en ese sentido cero rollo (Catalina, 38 años).

\section{La disposición para el tratamiento: la agencia dócil}

El proceso de tratamiento con las TRHA supone una experiencia -en ocasiones prolongada- de fuerte invasión corporal, de control periódico del cuerpo y en ocasiones de procedimientos que implican dolor físico. Esta experiencia exige de las mujeres usuarias cierta disposición y disciplinamiento para poder afrontar los diferentes momentos del proceso, especialmente en el inicio y en las primeras etapas del tratamiento, donde todo resulta desconocido.

Esta forma de acción dócil (Avisahi, 2008) no supone una actitud de pasividad en las mujeres, sino que aparece en los relatos como la disposición física y emocional necesaria para lograr un embarazo mediante las TRHA en determinados momentos.

Esta docilidad como una «condición para» permite disponerse física y emocionalmente a diversas instancias como la hormonización, la medicación, el control constante del cuerpo, la atención a la alimentación, el ejercicio físico, la superación de miedos. Así lo cuenta Candelaria, quien dispuesta al tratamiento no dramatizó el proceso sino que lo atravesó:

Me las pude poner [las inyecciones] porque (...) la verdad, desdramaticemos, hay gente que se hace quimioterapia y pierde el pelo, no sé, yo estaba como inyectada de vida, porque yo no suelo ser ni tan optimista (...), así que bueno, me pusieron unas inyecciones, estimulé los ovarios a un punto, y aparte leí por internet lo que tenía que comer, lo que tenía que hacer, todo lo que estimulaba, toda la alimentación que me ayudaba para que el organismo funcionara mejor, que la actividad física, que el agua con limón en ayunas, todo hice (Candelaria, 38 años).

El relato de Alcira también ilustra muy bien este tránsito de agencia dócil. Si bien se advierten características de fortaleza física y emocional frente al dolor, que resultan disruptivas frente a una mirada patriarcal que supone una usuaria frágil y pasiva, a la vez se da cuenta de una disposición que resulta necesaria para lograr el objetivo de su embarazo.

Era muy molesto, muy doloroso y muy molesto. Yo tengo un umbral alto del dolor y también soy muy orgullosa, entonces «¿te duele?», «no, no», entonces iba a trabajar como si nada. ¿Sí? Sí, no digo que sea bueno ni malo (Alcira, 63 años). 
Otros relatos que también dan cuenta de procesos prolongados con TRHA reflejan otras formas de disposición que se acercan más a la resistencia. Sandra que refiere que «se bancaba» los tratamientos, pero al igual que Candelaria lo hacía pensando en el fin, la maternidad. De alguna manera, esta docilidad combina resistencia y disciplina como parte de lo que había que hacer para lograr un embarazo, implica salirse de la esfera del conflicto y disponerse a lo que los médicos ordenaban. En este sentido, encontramos relatos de usuarias como Sofía, con una postura crítica hacia el sistema médico, la cual se convertía en un obstáculo para iniciar el tratamiento por no conformarse con ningún especialista:

Me acuerdo que salimos de uno, veníamos con uno que nos habían recomendado, habíamos hecho entrevista con un par más, y todavía nos quedaba un turno con una tercera, y [mi pareja] dice «Mirá, Sofía, a mí este me cayó bien, creo que ninguno nos va a caer del todo bien, ¿vos estás segura?». Bueno, le digo, no, «ya está». Nos quedemos con este y listo. Me atendió y después le iba diciendo, «mirá, re buena onda pero esto de que hiciste del parto de aquella cosa, a mí me parece que no va», o sea, bueno, le discutía cosas... (Sofía, 44 años).

Una vez superada esta primera fase, Sofía expresa que una vez establecida esta relación se permitía discutir y cuestionar las decisiones del médico, algo que no es común desde el lugar de paciente, como tradicionalmente se entiende desde una disposición de pasividad. Sin embargo, Sofía también señala que de igual manera «resignó cosas» porque sabía que era el requisito para poder avanzar con su tratamiento.

Pero como relata Brenda, hay algo de este proceso del tratamiento que tiene que ver con un proceso de aprendizaje. Por haber estado embarazada previamente, podía comparar algunas sensaciones de la hormonización con la sensación de estar embarazada, donde haber pasado por esa experiencia era un capital para sostener el tratamiento:

... entonces no es la misma ansiedad que manejaba, que alguien que estaba buscando un primer embarazo que en un segundo [embarazo]. Sí soy de pensar mucho «por algo será» (Brenda, 38 años).

Como señala el relato de Luz, quien al momento de su entrevista llevaba varios años de tratamiento, esta docilidad como disposición puede ser adquirida durante el proceso mismo con las TRHA. Lo que al principio del tratamiento se experimenta como molesto, doloroso, luego con su repetición se logra adquirir 
cierta disposición para afrontarlo así como una actitud de acostumbramiento. Por ejemplo, en relación a los medicamentos que se toman, las hormonas que se inyectan, el realizarse de forma permanente ecografías transvaginales:

Totalmente, ya no significa nada, ¿me entendés? Para mí, es más, a veces al final ya hasta le decía yo: «mejor me hago una este día y este día también» (...).Ya estás tan metida en el tema que ya no te significa nada, pero en ese momento sí significaba (Luz, 45 años).

Lo mismo relata Gisela, quien cuenta que antes del tratamiento tenía «miedo a las agujas». El hecho de tener que inyectarse cotidianamente hormonas Llevó a que aprendiera cómo hacerlo ella misma, superando este temor en pos del tratamiento. Al igual que en otros relatos se encuentran presentes en cada proceso los dolores físicos, molestias, sentimientos de invasión, estos van acompañados de una disposición diferente:

Nunca dije: «no, ¿por qué? O hasta aquí llego». Siempre fue: bueno, me va a doler, listo, pero vamos, es más, las primeras inyecciones que estábamos en casa, y al principio me ponía ahí mi marido, como que él también respiraba hondo y era: «bueno, pará, pará, pará, bueno, ahora, no, pará, pará, pará, listo, ponemelas», y hasta que arranqué y dije: «chau» (Gisela, 39 años).

El tránsito que asegura el «deseo» y la autoadministración de esta hormona, como señala Preciado (2008), supone toda una construcción y una disposición en las mujeres usuarias. Es decir, la autoadministración de la droga -las hormonas- no se produce automáticamente, sino que se aprende a desear. De esta manera, estos relatos, que a simple vistan suponen pasividad, también dan cuenta de un aprendizaje adquirido: es un aprendizaje disciplinante pero a la vez es la disposición necesaria que les permitirá agenciar su reproducción en el marco de esas relaciones de poder.

Por último, pensar esta agencia dócil supone no perder de vista que a la vez que las mujeres usuarias aprenden las disposiciones necesarias que les permiten soportar el malestar físico del tratamiento, también desarrollan un autocontrol, que como señala Preciado (2008) hoy se expresa en las formas en que el poder es introyectado.

\section{Transitando el tratamiento y gestionando las emociones}

Así como una actitud pragmática permite a las usuarias accionar sin mayores deliberaciones el inicio del tratamiento y la docilidad habilita los primeros 
aprendizajes, sostener el tratamiento -en ocasiones por años- requiere una adecuada «gestión de las emociones». Como señalan los relatos, los sentimientos de dolor y frustración pueden convertirse en un obstáculo para seguir con el tratamiento. Cuando se hace referencia a las disposiciones que se esperan de las mujeres en relación al tratamiento, no solo es una disposición física, sino que es toda una disposición psíquica y emocional que se sostenga en el tiempo. Esto es muy claro en el relato de Sabrina, a quien tras sucesivos intentos de inseminación artificial le aconsejaban que «no piense», «que se relaje» como cierta docilidad en relación al aspecto psicológico:

Una se siente como muy culpable también porque soy yo la que me embarazo y soy yo la que no me embarazaba, viste, es como que uno se echa mucho la culpa de ¿Por qué? ¿Qué? Y todo el mundo te dice: «bueno, no lo pienses, relájate», y vos decís: «¿Cómo no voy a pensar?» (...) estar en esa situación, es como que estás mal, estás triste, hay un montón de cosas que te están pasando que es imposible no pensar, digamos, te tenés que sacar el cerebro así y dejarlo a un costado para no pensar (Sabrina, 35 años).

La peculiar manera en que opera el poder y la ideología maternal sobre las mujeres se advierte claramente en esta reflexión de Sabrina. Por un lado, opera el mandato de la maternidad sobre las mujeres, pero paradójicamente cuando la maternidad se ve obstaculizada también se les demanda que no hagan de ello un conflicto, sino que aprendan a autogestionar este malestar. Las TRHA, desde la perspectiva del biopoder, podrían pensarse en el marco de nuevas formas en que opera el poder sobre los cuerpos a los que les exigen autocontrol de las emociones como una manera de transitar por el tratamiento y de que el mismo tenga éxito.

Otra forma de gestionar las emociones se vincula a la fragmentación corporal en las usuarias; aquí lo que aparece como disociado es la experiencia emocional de la corporal. La vivencia de la fragmentación del cuerpo se vincula con los sentidos occidentales de cuerpo y mente como entidades separadas, jerarquizadas, que se corresponden a la idea aristotélica de cuerpo humano (Maffía, 2005). Esta concepción en las experiencias con las TRHA se manifiesta mediante una idea de la psiquis separada del cuerpo, como entidades donde una domina a la otra, pero que a su vez permite separar las formas de padecimiento: el cuerpo es un lugar desde donde se puede soportar más sufrimiento que la mente.

Cuando las usuarias hablan del dolor y la frustración padecidos durante los tratamientos, la preocupación constante por el tratamiento supone, como bien señala Melina, un compromiso total con el proceso: 
... no deja de estar en tu cabeza, implica un compromiso total con el proceso (...) y con el tiempo vos lo vas, con información, con el tiempo, lo vas hablando con gente, es como que lo vas procesando de otra forma, no deja de estar presente pero lo procesás, pero no deja de estar en tus objetivos. De hecho, no deja de estar en mi cabeza; de hecho, pido todos los días cuando rezo, pido todos los días por lo mismo. Pero bueno, qué sé yo, tampoco deja de ser difícil, pero no es que lo tome con tanta naturalidad ni con tanta alegría, no deja de ser inquietante... (Melina, 41 años).

Y así también cuando se hace referencia al dolor físico, donde algunas entrevistadas relatan estudios muy dolorosos e invasivos, una constante de los relatos es que este dolor es contantemente comparado con otras formas de padecimiento emocional. El dolor físico pierde entidad cuando las mujeres usuarias lo comparan con aquello que vivencian como un sufrimiento psíquico o emocional:

No, en eso yo soy, a ver... a mí el tema del cuerpo, por más que hay cosas que sí son dolorosas, no me importan. A mí si me decís «te voy a pellizcar un montón, te voy a hacer, te voy a poner un gancho» (...) yo lo iba a hacer igual. No me importaba que me toquen el cuerpo, que me duela, no me importaba. A mí me afectaba más la cabeza, del tratamiento, yo con el cuerpo, decía «bueno» (Catalina, 38 años).

Uno de los sentimientos al que se refieren con frecuencia las usuarias que atraviesan tratamientos prolongados es la frustración por no obtener los resultados deseados con las TRHA. Gestionar este malestar implica a su vez un conjunto de estrategias que las mujeres despliegan para poder lidiar con el sufrimiento, en pos de que el mismo no se vuelva un obstáculo para sostener el tratamiento:

Es que no es poco porque vos tratás de que no te afecte pero te afecta igual porque cuando te ves imposibilitado, que no tenés los medios para hacerlo, te termina frustrando, y más allá de tu propia voluntad e intención, te condiciona, porque no está a tu alcance, entonces limitaciones que te frustran, y me hice todos los estudios porque muchos estudios y análisis clínicos se actualizan al mes... (Melina, 41 años).

El sufrimiento emocional podría revelar otro aspecto que se experimenta durante el tratamiento y que es difícil de sobrellevar. Esta idea de que el dolor 
físico es más soportable que el dolor emocional da cuenta de esta jerarquía de experiencias. Pero también supone la vivencia de que el cuerpo es más plástico y moldeable, mientras la mente es aquel aspecto que «condiciona» y obstaculiza. Es allí donde un conjunto de tecnologías del yo, psicológicas y espirituales, tienen lugar durante el tratamiento tan relevante.

Cuando este sufrimiento aparece poco autocontrolado por las usuarias es cuando comienza a verse de forma problemática por las instituciones biomédicas. En lugar de cuestionarse las maneras en que la medicina produce sufrimiento a las usuarias, el malestar es individualizado, lo que recuerda a las formas de histerización del cuerpo femenino que señala Foucault. Podría pensarse que aquello que se expresa como actitudes de ansiedad, obsesión o sentimientos de culpa son una de las maneras en que estas formas de control se manifiestan en sus subjetividades de forma individualizada. En este sentido, el acceso a la información, el conocer las posibilidades de «éxito o fracaso» constituyen una de las formas en que muchas usuarias aprendieron a «bajar la ansiedad» o a «no obsesionarse», dicho en sus palabras. La contracara de ello es que cuando el tratamiento no funciona, las usuarias tienden a revisar su conducta, vuelven sobre sus pasos para poder comprender la lógica mediante la cual disminuir los efectos la mencionada culpa:

A veces... «¿Qué habré hecho que no prendió?» «Qué habré hecho que no ovulé bien?», ¿me entendés? «QQué habré hecho mal?», porque es así. Aparecen todas esas cosas, (...) al seguir en un lugar [precisas que] te expliquen, te cuenten, qué te van a hacer, para qué te van a hacer la finalidad, que te digan las expectativas reales (Melina, 41 años).

Como señala Melina, saber las posibilidades reales de embarazo en cada tratamiento, contar con la información correcta y detallada de los tratamientos, ayuda al manejo de las propias expectativas, como una disposición que se aprende y adquiere, como tecnologías del yo (Rose, 2007).

Otra forma en que se expresa el trabajo de gestionar las emociones se vincula a la manera en que las usuarias resuelven la duda por el origen de la llamada «falla reproductiva»: ¿es un problema físico o psicológico? Esta pregunta se vuelve tramposa para las usuarias, dado que en el marco de una pareja heterosexual donde el diagnóstico de «infertilidad» es la etiqueta, tiene efectos de responsabilización diferenciales el lugar donde su ubique esta falla.

Pensar su situación desde la infertilidad, como una «enfermedad», si bien tiene efectos patologizantes sobre sí, en el relato de Gisela aparece como una manera de nombrar este obstáculo de una forma que no la hace sentirse 
culpable. De esta manera, la infertilidad, entendida como una «enfermedad», como algo «que le pasa» a las personas más allá de su voluntad, desculpabiliza y a la vez permite encontrar recursos y respuestas:

¿Vos lo viviste así o lo...? [en referencia a que Gisela habla de infertilidad].

G: Me costó darme cuenta pero sí. Siempre me costó y siempre decía: «ipero por qué?» Yo prefiero que me encuentren algo y que me digan "estás enferma, tienes tal problema, y hay tal cura», a que me digan «bueno, no, es la mente, seguí esperando, hacé esto, hacé lo otro» (Gisela, 39 años).

Si bien la categoría de infertilidad es una noción estigmatizante y heterocentrada (Farji Neer, 2015; Viera Cherro, 2012), ciertas identidades como la de «infértil» no solo actúan como estigmas sino que generan nuevas formas de biosociabilidad y permiten agenciar a las personas desde estas nuevas identidades estratégicas (Rose, 2007). Ello se puede visualizar en el relato de Gisela, la única entrevistada que define su situación como «infertilidad». Ella no utiliza esta categoría desde un lugar de identificación sino desde las posibilidades de acción que ofrece una sociedad medicalizada. Posicionarse desde el lugar de «infértil» es estigmatizante, pero a su vez le permitió resistir la idea de que la causa de su dificultad está asociada a una causa psicológica y actuar en consecuencia con los recursos de la medicina.

Partiendo de que las mujeres usuarias son agentes que reconocen sus obstáculos y agencian en consecuencia con las posibilidades que le ofrece la sociedad, el mercado y la tecnología, para algunas mujeres estas categorías estigmatizantes resultan la única manera de nombrar y resolver la voluntad de tener hijos. Aun a riesgo de quedar atrapadas en las TRHA como única vía legitima para lograr la maternidad, estas les otorgan inteligibilidad a su demanda y habilitan con todas sus restricciones y posibilidades su agencia reproductiva.

Así mismo, estos relatos dan cuenta de que hay una resistencia en las usuarias a ser patologizadas, y en este sentido es interesante cómo las usuarias entienden que es preferible que sea el cuerpo -vivido como ajeno- el etiquetado como «infértil» a que sea un problema psicológico, el cual se identifica culturalmente con la propia personalidad.

Por otra parte, Catalina relata que se resistía en consultar a un/a psicóloga/o por esta causa, ya que desde su perspectiva implicaba «darse por vencida» o asumir la «infertilidad»: «... ¿qué le voy a contar? Quiero ser mamá. Sí, iba a ir al psicólogo el día que hubiese llegado de hacer diez tratamientos, yo iba a ir para asumir que no iba a ser mamá si no lo podía ser» (Catalina, 38 años). Ir 
al psicólogo para Catalina va en el mismo sentido que para las demás usuarias: querer ser madres no es una decisión problemática, lo problemático es la infertilidad, en consecuencia sus esfuerzos están orientados a resolver los obstáculos -físicos- que encuentran para tener hijos.

\section{Tomando el control del tratamiento: migraciones y cambios}

Las trayectorias de las mujeres por las instituciones -clínicas, médicas, jurídicas, obras sociales-durante el tratamiento, por un lado evidencian la cantidad de tareas que forman parte de este trabajo reproductivo, pero también constituyen momentos de decisión y de cuestionamiento de la autoridad médica que las llevan a cambiar de decisión sobre el rumbo del tratamiento. En este transitar las usuarias se posicionan estratégicamente desde múltiples identidades: pacientes, clientes, usuarias, titulares de derechos, como identidades que posibilitan en determinados contextos y de forma simultánea su agenciamiento (Rose, 2007). Es por eso que las maneras en que las mujeres transitan por los espacios médicos, como trazos de cartografías (Gual Martí, 2011), permiten dar cuenta de otras formas de actuar en estos espacios biomédicos más cercanas a la crítica y a la disputa.

Una forma en que las usuarias se enfrentan con el malestar en las instituciones medicas es mediante las llamadas «migraciones» por las propias usuarias. Esta categoría da cuenta de estrategias desplegadas ante el trato inadecuado de los médicos, ante la falta de contención, información o frente a un tratamiento en donde perciben no tendrán resultados efectivos. Así, Sofía señala que al no tener resultados positivos en la primera parte del tratamiento, por ejemplo, y por no acordar con algunas decisiones médicas, decide migrar de la clínica donde había comenzado el tratamiento:

Y entonces, eso, fracasado eso tres o cuatro veces (...) [escucha que la médica le dice a su pareja] «no salió ningún óvulo, no pudimos recuperar ningún óvulo...», ella dijo que visto este resultado, ella proponía la ovodonación. Y como había algunas cosas de la atención que no me habían gustado, yo creo que esta mujer sabía muchísimo (...) Entonces no tenía quirófano, teníamos que ir a un quirófano de otro lugar, lugar muy desconocido y todo eso a mí me sonaba como mal. Entonces dije «¿sabes qué? Vamos a cambiar de lugar» (Sofía, 44 años).

Esta estrategia de las mujeres que las posiciona como usuarias en el marco de una red de oferta reproductiva cada vez mayor en la ciudad de Córdoba forma parte del repertorio de acciones que les permiten elegir y decidir en 
el marco de sus recorridos. Así lo relata Melina, quien hace referencia a estos «cambios y migraciones»:

Me fui a otro instituto. En realidad fue por eso. Quizás el fracaso. Que tampoco es responsabilidad del médico, o total responsabilidad del médico. Pero me dio bronca y hablar con otras chicas, (...) una conocida de una amiga mía le contó que había ido primero a [nombre de clínica 1] y que no le había gustado como la trataban, después se fue a [nombre de clínica 2], hizo el procedimiento completo y quedó embarazada, y yo le digo «[nombre de su pareja], ¿qué hacemos?». «¿Querés cambiar? Cambiemos». «¿Y si es lo mismo?» «Y cambiá» (Melina, 41 años).

Particularmente algunas mujeres señalaban que en algunas clínicas experimentaron un límite en el tratamiento así como un fuerte desánimo, donde las opciones se iban reduciendo cada vez más. De esta manera, cambiar de centro permite no solo pensar en nuevas opciones, sino que constituye una contestación al diagnóstico médico que las culpabiliza frente a su dificultad reproductiva:

Y es por esta cuestión que a uno le cuesta asumir ese porcentaje de que no se dé, ¿me entendés? Ese porcentaje de posibilidades de que no se dé, a uno le cuesta asumir, capaz hacer responsable al instituto. Y quizás no es el instituto. Sos vos, o es el destino, yo también creo mucho en eso del destino, que las cosas se dan porque ya están señaladas (...) por eso la migración, «vamos acá, probemos acá, vamos acá, probemos acá, volvemos acá, probemos acá» (Melina, 41 años).

De alguna manera, estos recorridos y «migraciones» se vinculan a este tipo de agencia pragmática, orientada a resolver. Pero al mismo tiempo permite a las mujeres y sus parejas ponerse en el centro de las decisiones, y pone en evidencia la importante determinación que tienen las mujeres usuarias en el marco de este tratamiento:

Para mí, el médico era un medio para lograr mi objetivo, nada más. Por eso cuando no lograba el objetivo con este médico, me recomendaban uno mejor, buscaba otro. No hacía yo ningún lazo afectivo ni de dependencia con ninguno. Me servía o no me servía para lo que yo quería y nada más (Sandra, 44 años).

De esta manera, si bien a simple vista pareciera que las mujeres transitan por escenarios medicalizados donde su voz no es oída, también se puede advertir que esta agencia es posible por algunas fisuras vinculadas a otras 
lógicas de poder. Esto quiere decir que este tipo de agencia se vincula a aquellas disposiciones que se adquieren en el marco de un mercado reproductivo donde las mujeres usuarias no solo son pacientes, sino también consumidoras que exigen la efectividad en los servicios de salud (Rose, 2007). Ello supone reforzar el lugar de elección que ponen en juego «ethos individualizantes» (Duarte, Jabor, Gomes y Luna, 2008) donde los derechos son titularidad del individuo.

\section{La apropiación del proceso: el aprendizaje del lenguaje médico}

El lenguaje médico sobre los cuerpos reproductivos puede ser analizado desde una doble relación a partir de las experiencias reproductivas: como un discurso ajeno, incomprensible, que distancia a las mujeres de sus procesos reproductivos, pero a la vez como un discurso apropiado e incorporado en sus relatos por la experiencia adquirida en el tratamiento. Como señala Emily Martin (2001), el hecho de que las mujeres utilicen los términos de la ciencia dominante para hablar de sus procesos corporales reproductivos da cuenta de que la hegemonía médica no se construye por fuera de las propias subjetividades sino que aparece como sentido común en sus relatos.

Algunas usuarias señalan al lenguaje médico como un obstáculo inicial para comprender lo que estaba sucediendo en su tratamiento. Esto obedecía tanto a dificultades para comprender los términos con los que se habla de sus cuerpos y sus procesos reproductivos como así también a no entender los efectos que tenían en su tratamiento un resultado u otro. Esta cripticidad del lenguaje aparece en el relato de Candelaria cuando expresa su inseguridad inicial sobre sus conocimientos sobre el propio cuerpo, así como las formas de nombrar los procesos reproductivos. Con relación a la menstruación, Candelaria relata que no podía identificar cuál era el que los médicos llamaban «día uno», fecha que para ella era ambigua y tenía otros sentidos y consecuencias en su vida cotidiana:

Bueno, ese, ese es feo, después trasvaginales, todas que te coincidan con determinados días del período, el día dos, el día siete, el día ocho, hubo un mes que suspendí todo porque me entró la locura de cuál era el día uno del período (Candelaria, 38 años).

Así también, el desconocimiento del lenguaje médico le provocó a Catalina una gran frustración en los primeros tratamientos. Por ejemplo, cuando escuchaba el relato de los médicos sobre los procedimientos realizados, interpretaba que los mismos habían sido exitosos. Descubrir la situación contraria 
generó en ella una primera experiencia negativa no solo por los resultados, sino además porque por su desconocimiento no había previsto esta situación:

... encima como uno no entiende empiezan ellos a decir las medidas de los óvulos, entonces, bueno, hay gente que le funciona bien y terminan sacando diez óvulos (...) y yo escuchaba muchos números, decía guau, cuántos, y capaz que eran tres nomás, y decía «qué bárbara que estoy», «qué divina», y cada vez que iba y eran medidos y «están re grandes» (...) y salgo de ahí feliz con mi esposo, «acá nos dicen que mañana nos hacen el tratamiento», entonces entro re feliz y él me dice «chicos, no está dando resultado. Yo no seguiría porque esto no funciona, así que estaría bueno suspenderlo e iniciarlo en otro ciclo». Ah, casi me muero, ese día era como si me hubiesen matado un hijo vivo, (...) así que eso fue para mí, muy extraño (Catalina, 38 años).

De alguna manera, este proceso le exigió a Catalina aprender este lenguaje médico para poder suavizar el impacto de la información en el proceso, durante un tratamiento prolongado: «Y después, cuando te va pasando muchas veces esto, decís "uy, otra vez", o sea, siempre frustrante, pero creo que esa vez... [haciendo referencia al malentendido en el procedimiento]» (Catalina, 38 años).

Del mismo modo, el relato de Rosa, quien nombra las técnicas con un lenguaje médico en su relato, podría pensarse como resultado de un proceso de aprendizaje en el marco de una experiencia de gran malestar en la clínica, que se vinculaba entre otros aspectos a la poca información que le brindaban los médicos sobre el proceso: «Sí, necesitaba eso, necesitaba una comunidad que me explicara un poco qué era lo que iba a venir, porque los médicos no lo hacían. Yo por ahí les preguntaba un paso más adelante y ella [la médica] me decía: "no, futurología yo no"» (Rosa, 37 años).

Por otra parte, si bien la mayoría de las usuarias se caracteriza por contar con un capital cultural y educativo que les facilita el aprendizaje y comprensión de procesos, aun así este lenguaje les resultaba ajeno a sus propias experiencias corporales. Podría pensarse que el aprendizaje, así como la resistencia al lenguaje médico, introduce a las mujeres en un campo donde la violencia simbólica opera de tal manera que estas aprenden a nombrarse de acuerdo a los términos impuestos por la medicina -«infértil»-, pero a la vez también habilita posibles reposicionamientos y aprendizajes sobre las maneras en que se habla de ellas.

Una de las maneras en que la violencia se hace presente en los relatos tiene que ver con el modo en que las mujeres hacen uso de una terminología 
mercantilista y eficientista del lenguaje biomédico: se hace referencia a la «calidad» y al «rendimiento» de los cuerpos y de las gametas de las mujeres.

Como se señaló anteriormente, este lenguaje es apropiado por las mujeres para explicar sus obstáculos reproductivos, como una manera de comprender los resultados de los tratamientos y para tomar decisiones durante el mismo. Pero este proceso de apropiación del lenguaje, en lugar de posibilitar la subversión de este estigma, en este caso lo refuerza, naturalizando concepciones que sostienen que las mujeres pierden valor cuando pierden su capacidad de gestación. Esta mirada biologicista de los cuerpos y la reproducción opera mediante la jerarquización de los cuerpos desde la intersección de la edad y el género, aspecto que se reproduce constantemente en la experiencia de las mujeres usuarias.

A través del vocabulario productivista, es frecuente que las usuarias hablen de la «calidad» y la «edad» de sus ovocitos, como una explicación al fracaso de un tratamiento, o las causas por las que deciden recurrir a la ovodonación.

Como se puede ver en los relatos de María y Luz, las nociones de «calidad» y «edad» empiezan a ser usados como sinónimos. El concepto de calidad aparece a su vez fuertemente naturalizado en sus relatos:

El problema es la mujer porque nosotras nacemos con unos óvulos y no se reproducen; es esa cantidad, esa reserva ovárica, digamos. La cantidad de óvulos es con la que nacemos y la calidad es la edad de la mujer. Hoy, al menos, no hay algo que te diga, un estudio para hacer más que el embrión que lográs cuando hacés el [procedimiento] in vitro. Ahí [recién] ellos ven la calidad del embrión... (Luz, 45 años).

Pero esta neutralidad científica no es tal, sino que forma parte de un lenguaje que tiene efectos de género. En este sentido, las usuarias son conscientes de que este lenguaje hace referencia a ellas mismas, y así lo incorporan en sus explicaciones, tal como hace María:

Y contra eso no se puede hacer mucho (...) Me inyectaron lo más de lo más. O sea, lo último de lo último, y mis ovarios ni se inmutaron, no produjeron ni un folículo, entonces fueron ensayando y probando, con menos cantidad, algo más tranqui, y pudieron extraer en alguna punción, dos, tres, pero ya eran de baja calidad, porque tus folículos tienen tu edad, ya no son de una chica de dieciocho, diecinueve. Ya tenía, cuando empecé tenía treinta y ocho, treinta y nueve.

E: Qué fuerte eso de calidad. ¿Son palabras que usan ellos? 
M: Sí, porque tienen un ranking, se los clasifica a los ovocitos, cuando te lo extraen te dicen, tipo uno, tipo dos, tipo tres, tienen una nomenclatura (María, 43 años).

Esta manera de entender a los ovocitos como recurso escaso, que afecta espacialmente a las mujeres, puede asociarse con lo que Emily Martin (1991) identifica como aquellos sentidos culturales negativos que circulan sobre la menstruación y el cuerpo de las mujeres desde el lenguaje médico. De este modo, las usuarias se apropian del lenguaje, adoptan esta representación sobre sus cuerpos, como una forma de dar sentido a su experiencia reproductiva. Este lenguaje a su vez, si bien estigmatizante, le permite a María explicar con claridad las razones por las cuales no logró un embarazo después de varios tratamientos:

Y después, cuando decido pasar al in vitro, ahí los análisis ya me salían mal, porque yo tenía una reserva ovárica muy baja... que eso si yo lo hubiera empezado antes capaz lo hubiera obtenido, cuando te extraen folículos, te hubieran extraído más (...) nosotras venimos con una carga ovárica determinada, algunas vienen con mucho y otras con poca (María, 43 años).

Si bien pocas usuarias hacen referencia a la infertilidad como diagnóstico, los términos de «baja calidad», «baja reserva ovárica», «envejecimiento» son diferentes nociones que están conectadas a la misma. Esta reapropiación del lenguaje médico habilita a nombrar aquello que se vive como un fracaso, explicarlo, aun cuando los términos para hacerlo sean estigmatizantes. Estas formas de nominarse forman parte de este aprendizaje que se produce a lo largo del trayecto mismo con las TRHA, como se analizó en estas últimas formas de agencia, y que solo son posibles por estar inmersas en esas relaciones de poder, desde la perspectiva de Judith Butler.

\section{La trayectoria final y la agencia cuestionadora}

Como se pudo analizar, en el accionar de las mujeres usuarias por el transito en las clínicas conviven diferentes disposiciones: en ocasiones los tratamientos exigen cierta agencia dócil, acorde a un modelo de medicina del siglo XIX, que supone cierto sujeto-paciente. Al mismo tiempo, el escenario biomédico actual también exige otras disposiciones, como consumidoras, ciudadanas, como agentes que se autoadministran las hormonas y hacen propia la vigilancia (Preciado, 2008), y que hasta disputan la direccionalidad del tratamiento de ser necesario. 
Este tipo de agencia se advierte con claridad al final del proceso reproductivo particularmente ante situaciones que las usuarias visualizan que pueden poner en riesgo el tratamiento. Aquí las usuarias dotadas de algunos saberes aprendidos en el marco de su recorrido toman el control de la tecnología mediante el acceso a medicamentos, hormonización, tratamientos para lograr sus objetivos. Para ello debieron pasar por diversos tratamientos de forma dócil, aprender a usar las tecnologías, asimilar las inyecciones, las múltiples ecografías y contar con nueva información. Como señala Franklin (1997), una vez pasado el primer ciclo se gana en un mayor sentido de control sobre el tratamiento, como parte de este aprendizaje.

De esta manera, encontramos relatos como el de Sofía, con vastos recursos educativos para cuestionar las decisiones médicas, quien da cuenta de estas diferentes formas de disponerse y vincularse con la medicina en diferentes momentos del proceso. Así como se analizó en su relato cierta agencia dócil al inicio del tratamiento, al final de su tratamiento pudo sostener otros reposicionamientos frente al saber médico. Por ejemplo, en cuanto al vínculo entre lactancia y embarazo, ella relata una conversación donde enfrenta las decisiones de su médico que le había aconsejado no amamantar durante el embarazo y ella lo contradice con conocimientos sobre lactancia.

Así también, María, quien relata una relación ambivalente con la ciencia tanto por sus creencias religiosas como por su formación educativa amplia, es una usuaria que no confía ciegamente en la medicina reproductiva, sino que cuestiona, pide explicaciones y prueba otras alternativas. En este sentido, ella cuestiona la ausencia de un diagnóstico claro durante el proceso frente a las repetidas pérdidas de embarazos, donde ser categorizada como una paciente de «causa dudosa» no constituía para ella una respuesta:

Sí, totalmente. Al contrario, me enojaba y me decían... el doctor me intentaba explicar: «la ciencia no tiene respuesta a todo por más que a veces diga que la tiene». Hay un gran margen de casos que nosotros no logramos, que son de causa dudosa. Por ejemplo, por qué las mujeres abortan, y bueno, en un $30 \%$ se puede explicar por qué abortan y en el resto no. Hay más cantidad de casos que son inexplicables... (María, 43 años)

Así como algunas usuarias, una vez comenzado el tratamiento reproductivo, relatan cierta agencia dócil a la hora de enfrentar el mismo, también dan cuenta de otras acciones posteriores que les permitieron tomar o negociar cierto control sobre el proceso reproductivo. Por ejemplo, la consulta con dos médicos de forma paralela es otra estrategia que aparece en uno de los relatos. 
Tal es el caso de Catalina, quien se realizó un tratamiento por trombofilia de forma paralela al tratamiento de fertilidad que se realizaba con el médico de la obra social; se lo ocultaba a este porque temía que revelar esta situación obstaculizara el tratamiento y cobertura:

C: Probamos algo, y no sale... Yo soy como que estoy en algo y de mmm, no, acá hay que probar otra cosa. Yo no sabía qué era lo que [sucedía], entonces yo pruebo algo y no funciona y digo «hagamos más». No me quedo, y ahí nomás busco otra cosa (Catalina, 38 años).

Si bien Catalina había sido una paciente respetuosa de las decisiones médicas durante el tratamiento, estaba en riesgo su deseo y la posibilidad de que este último intento fuera cubierto por la obra social, lo que la llevó a encarar otra estrategia que, cabe señalar, permitió a Catalina embarazarse en esa ocasión:

Pero... acá está [señala a su hijo en la falda], me funcionó [se ríe]. Yo ya sé que no se le oculta información al médico, pero yo sabía que ahí me faltaba algo, y ellos nunca supieron. Ay, la desesperación de andar con las inyecciones en la panza, «si ellos me las ven». No, nunca pasó nada... (Catalina, 38 años).

Es así que, como señalamos anteriormente, la propia autoadministración de las hormonas permite resquebrajar no solo la autoridad médica, sino que este autocontrol del proceso reproductivo hace que mujeres usuarias como Catalina se pongan a la cabeza de su tratamiento, lo que en este campo medico jerarquizado la corre del lugar de «paciente», con todo lo que ello implica como transgresión en este espacio. Sin embargo, la agencia de las usuarias no solo se expresa en estos casos de disputa explícita, sino que a lo largo de todo el recorrido es posible hablar de diferentes formas de agencia como formas de acción posibles durante el tratamiento.

\section{Conclusiones}

A lo largo del itinerario del tratamiento con TRHA fue posible advertir diversas formas de agenciamiento, que ponen en discusión la total objetivización de las usuarias en el marco de la medicina reproductiva. Al iniciar el proceso, se advierte una disposición de «docilidad» y «pragmatismo» en las usuarias, como una manera de asumir un proceso que ocasiona incomodidad, malestar y dolor físico. A lo largo del mismo se advierte que las usuarias despliegan un proceso 
de «gestión de las emociones» que les permite cierto autocontrol frente a este malestar vivenciado. Otra disposición se vincula al aprendizaje y apropiación de un leguaje médico hasta ese momento desconocido sobre el proceso reproductivo para comprender la lógica del tratamiento y tomar decisiones. Es a partir de este proceso y como consecuencia del aprendizaje realizado que en instancias más cercanas al final del trayecto las usuarias toman el control sobre el rumbo del tratamiento, siendo críticas con las clínicas y cuestionando las decisiones médicas de ser necesario.

De esta manera, estos procesos dan cuenta que las diferentes formas de agencia de las usuarias son posibles en el marco del propio campo biomédico. De alguna manera, las TRHA, como tecnologías de género (De Lauretis, 1996), por momentos habilitan ciertas fisuras no esperadas, así como se producen otras formas de habitar el control medicalizado que no necesariamente se expresan como resistencia u oposición al poder, sino que se advierten en otras modalidades. Por el contrario, implica que las usuarias desarrollen cierta adhesión y apropiación del tratamiento, disposiciones que son las que permiten luego negociar, cuestionar y transformar estas relaciones. Para quien se propone comprender las experiencias singulares de las usuarias, también suponen un desafío, ya que incitan a cuestionar las miradas dicotómicas que la academia propone sobre las tecnologías reproductivas y comprender la complejidad de este campo en movimiento.

\section{Notas}

1. De acuerdo a las OMS, la infertilidad es una «enfermedad del sistema reproductivo definida como la incapacidad de lograr un embarazo clínico después de doce meses o más de relaciones sexuales no protegidas». « VOLVER

2. Se recabaron experiencias sobre aquellos tratamientos disponibles y actualmente legales en Argentina, entre los que se diferencian los de «baja complejidad», como la inseminación artificial (con donantes de esperma u ovocitos) y los de alta complejidad, como la fecundación in vitro y la inyección intracitoplasmática de espermatozoide (ICSI), también recabando experiencias con donación de gametos y con gametos propios. « VOLVER

3. La identidad de las usuarias entrevistadas ha sido resguardada respetando el acuerdo de confidencialidad. « VOLVER 


\section{Referencias bibliográficas}

AHMED, S. (2015). La política cultural de las emociones. México: Universidad Nacional Autónoma de México, Programa Universitario de Estudios de Género.

ARIZA, L. (2014). La construcción narrativa de la infertilidad. Mujeres que narran la experiencia de no poder concebir. Sexualidad, Salud y Sociedad. Revista Latinoamericana, 18, 41-73.

ARMSTRONG, D. (1995). The Rise of Surveilance Medicine. Sociology of Health \& Illness, 17(3), 393-404. Disponible en: https://doi. org/10.1111/1467-9566.ep10933329

AVISHAI, O. (2008). «Doing Religion» In a Secular World. Gender \& Society, 22(4), 409-433. Disponible en: https://doi. org/10.1177/0891243208321019

BOURDIEU, P. (2005). La práctica de la sociología reflexiva En: Bourdieu, P. y Wacquant, L. Una invitación a la sociología reflexiva. Buenos Aires: Siglo XXI Editores. BOLTANSKI, L., y Thévenot, L. (2000). The Reality of Moral Expectations: A Sociology of Situated Judgement. Philosophical Explorations: An International Journal for the Philosophy of Mind and Action, 3(3), 208-231. Disponible en https://doi. org/10.1080/13869790008523332

BURY, M. (1982). Chronic Illness as Biographical Disruption. Sociology of Health and Illness, 4(2): 167-182.

BUTLER, J. (1997). Lenguaje, poder e identidad. Madrid: Síntesis. Disponible en: https://doi. org/10.1017/CBO9781107415324.004

CARRANZA OSORIO, R. M. (2017). El significado del diagnóstico en la trayectoria del enfermo reumático: De la incertidumbre a la disrupción biográfica. Salud colectiva, 13, 211-223.

COREA, G. (1985). The Mother Machine: Reproductive Technologies from Artificial Insemination to Artificial Wombs. Nueva York: Harper Collins.

CORNEJO, M.; Mendoza, F., y Rojas, R. C. (2008). La investigación con relatos de vida: Pistas y opciones del diseño metodológico. Psykhe, 17(1), 29-39. Disponible en: https:// doi.org/10.4067/s0718-22282008000100004

CONRAD, P. (1992). Medicalization and Social Control. Annual Review of Sociology, 18(1), 209-232.

CROWE, C. (1985). «Women Want It»: In-vitro Fertilization and Wwomen's Motivations for Participation. Women's Studies International Forum, 8(6), 547-552. Disponible en: https://doi.org/10.1016/0277-5395(85)90092-5

DE LAURETIS, T. (1996). La tecnología del género. Revista Mora 2, 6-34. Duarte, L. F. D.; Jabor, J. de M.; Gomes, E. C., y Luna, N. (2008). Family, Reproduction and Religious Ethos in Brazil. Subjectivism and Naturalism as Structuring Values. Archives de Sciences Sociales Des Religions, 53(142), 113-130. Disponible en: https://doi. org/10.2307/30116918

FARJ NEER, A. (2015). Cuerpo, derechos y salud integral: Análisis de los debates parlamentarios de las leyes de Identidad de Género y Fertilización Asistida (Argentina, 20112013). Salud Colectiva, 11(3), 351-365. Disponible en: https://doi.org/10.18294/ SC. 2015.721

FRANKLIN, S. (1997). Embodied Progress. Londres: Routledge. 
FRANKLIN, S. (2009). Transbiology: A Feminist Cultural Account of Being After IVF. Critical Conceptions: Technology, Justice, and the Global Reproductive Market. Barnard U.

GofFMAN, E. (2001). Internados. Buenos Aires: Amorrortu.

GUAL MARTí, A. (2011). Maternidad y Técnicas de Reproducción Asistida: Un análisis, desde la perspectiva de género, de los conflictos y experiencias de las mujeres usuarias. Universitat Jaume I.

HARAWAY, D. J. (1999). Las promesas de los monstruos: Una política regeneradora para otros inapropiados/ble. Política y Sociedad, 30.

LIBSON, M. C. (2012). Parentalidades gays y lesbianas: los cambios de relato. Sociedade e Cultura, 15(2), 309-317.

MAfFía, D. (2005). El contrato moral. En Carrió, E. y Maffía, D. (ed.), Búsquedas de sentido para una nueva política. Buenos Aires: Paidós.

MAHMOOD, S. (2001). Feminist Theory, Embodiment, and the Docile Agent: Some Reflections on the Egyptian Islamic Revival. Cultural Anthropology, 16(2), 202236. Disponible en: https://doi.org/10.1525/ can.2001.16.2.202

MAHMOOD, S. (2008). Politics of Piety: The Islamic Revival and the Feminist Subject by Saba Mahmood. PoLAR: Political \& Legal Anthropology Review, 31. Disponible en: https://doi.org/10.1111/i.1555$\underline{2934.2008 .000272 . x}$

MARTIN, E. (2001). The Woman in the Body. A Cultural Análisis of Reproduction. Boston: Beacon Press.

MENÉNDEZ, E. L. (2003). Modelos de atención de los padecimientos: de exclusiones teó- ricas y articulaciones prácticas. Ciência \& saúde coletiva, 8, 185-207.

MENDES DIZ, A. M.; Domínguez Mon, A., y Schwarz, P. K.N. (2011). Enfermedades crónicas. Entre la autonomía y el control. IX Jornadas de Sociología. Facultad de Ciencias Sociales, Universidad de Buenos Aires, Buenos Aires.

Mercado-Martínez, F. J.; Robles Silva, L.; Ramos Herrera, I. M.; Leal, N. M., y Alcántara Hernández, E. (1999). La perspectiva de los sujetos enfermos. Reflexiones sobre pasado, presente y futuro de la experiencia del padecimiento crónico. Cadernos de saúde pública, 15(1), 179-186.

MoRGAN, L. M. (2019). Reproductive Governance, Redux. Medical Anthropology, 38(2), 113-117. Disponible en: https://doi.or g/10.1080/01459740.2018.1555829

PetCHESKY, R. P. (1987). Fetal Images: The Power of Visual Culture in the Politics of Reproduction. Feminist Studies, 13(2), 263-292. Disponible en: https://doi. org $/ 10.2307 / 3177802$

PRECIADO, B. P. (2008). Testo Yonqui. Madrid: Calpe.

RAPP, R. (1999). Testing Women, Testing the Fetus: The Social Impact of Amniocentesis in America (Anthropology of Everyday Life). New York, United States: Routledge. ROSE, N. (2007). The Politics of Life It Self. Biomedicine, Power and Subjectivity in the Twenty First Century. Oxford: Princeton.

ROSTAGNOL, S. (2015). Los cuerpos del aborto. Revista Venezolana de Estudios de La Mujer, 20(44), 163-177.

StRATHERN, M. (1992). Reproducing the Future. Anthropology, Kinship, and the New Reproductive Technologies. Mánchester: Manchester University Press. 
StraW, C. (2013). Cuando las demandas al Estado permanecen en el ámbito privado, íntimo y personal: el caso de la reproducción asistida para mujeres de sectores populares y medios, Área Metropolitana de Buenos Aires, X Jornadas de Sociología. Facultad de Ciencias Sociales, Universidad de Buenos Aires, Buenos Aires 0-8.

STOLCKE, V. (1986). New Reproductive Technologies - Same Old Fatherhood. Critique of Anthropology, 6(3), 5-31. Disponible en: https:// doi.org/10.1177/0308275X8600600302
THOMPSON, C. (2005). Making parents. The Ontological Choreography of Reproductive Technologies. Londres: MIT Press Books.

VIERA CherRo, M. (2012). Inequidades múltiples y persistentes en el campo de la reproducción asistida. Revista de Antropología Social, 21, 251-271.

WALDBY, C., y Cooper, M. (2010). From Reproductive Work to Regenerative Labour: The Female Body and the Stem Cell Industries. Feminist Theory, 11(1), 3-22. Disponible en: https://doi.org/10.1177/1464700109355210 